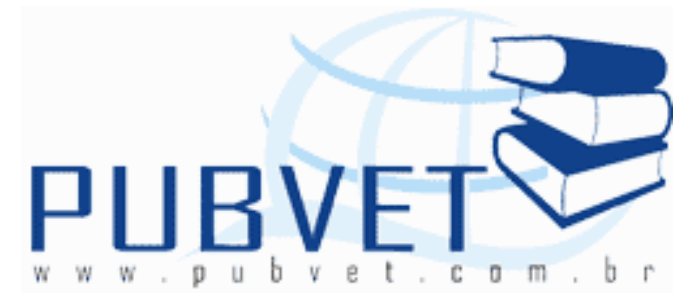

PUBVET, Publicações em Medicina Veterinária e Zootecnia.

\title{
Qualidade de carcaça e carne de frangos com uso de glicerina na alimentação
}

Peter Bitencourt Faria ${ }^{1}$; Carlos Henrique de Figueiredo ${ }^{2}$; Rosilene Silva Lima ${ }^{3}$; Douglas Batista Nascimento ${ }^{3}$; João Marcos Novais Tavares ${ }^{3}$; Cinara de Cássia Sousa Santos ${ }^{4}$; Ana Maria Barcellos Guerra Pinto ${ }^{4}$; Jorge Luis da Silva ${ }^{2}$

${ }^{1}$ Professor da Universidade Federal de Lavras, Departamento de Medicina Veterinária, Caixa Postal 3037, CEP 37200-000, Lavras-MG, Brasil. E-mail: peterbfvet@yahoo.com.br, Telefone: (35) 3829-5201.

2 Professor do Instituto Federal de Educação, Ciência e Tecnologia de Mato Grosso- IFMT, Campus São Vicente, BR 364, KM 329, CEP 78106-970, Santo Antonio do Leverger-MT, Brasil.

${ }^{3}$ Graduando do curso de Zootecnia do Instituto Federal de Educação, Ciência e Tecnologia de Mato Grosso- IFMT, Campus São Vicente, BR 364, KM 329, CEP 78106-970, Santo Antonio do Leverger-MT, Brasil.

${ }^{4}$ Graduando do curso de Medicina Veterinária da Universidade Federal de Lavras, Departamento de Medicina Veterinária, Caixa Postal 3037, CEP 37200000, Lavras-MG, Brasil.

\section{Resumo}

O objetivo deste estudo foi avaliar a alterações nos parâmetros de carcaça, qualidade e composição centesimal da carne de frangos alimentados com dietas com diferentes níveis de glicerina bruta. Foram utilizados 105 frangos 
machos da linhagem Cobb, criados em sistema intensivo com fornecimento das dietas a partir da fase inicial até a terminação, com abate aos 42 dias. 0 delineamento foi inteiramente casualizado (DIC) com cinco tratamentos e sete repetições $(5 \times 7)$, sendo cada parcela experimental constituída por três aves. Os tratamentos foram os seguintes: T1 - ração basal sem adição de glicerina; T2 - $30 \mathrm{~g} / \mathrm{kg}$ de adição de glicerina; T3 - $60 \mathrm{~g} / \mathrm{kg}$ de adição de glicerina; T4 $90 \mathrm{~g} / \mathrm{kg}$ de adição de glicerina e T5 - $120 \mathrm{~g} / \mathrm{kg}$ de adição de glicerina. Os resultados mostraram efeito dos níveis de glicerina sobre os parâmetros de carcaça (Peso ao abate, Rendimento de carcaça e do Peito, Peso das Pernas e Asas). Para a cor da carne do peito, ocorreu aumento dos teores de vermelho e alteração no ângulo de tonalidade da cor. Não foram observadas alterações em relação à perda de peso por cozimento, pH final e maciez em ambos os cortes. A glicerina bruta não promoveu alteração nos valores de composição nutricional na carne de frango independente do nível de utilização e do corte. De forma geral, a glicerina bruta na alimentação de frangos apesar de afetar os parâmetros de carcaça, destaca-se como um ingrediente de potencial uso devido às reduzidas alterações observado em relação à qualidade de carne.

Palavras-chave: maciez, cor, produção animal, nutrição

\section{Carcass and meat quality broilers with the use of glycerin}

\section{Abstract}

The objective of this study was to evaluate changes in the parameters of carcass quality and composition of meat from chickens fed diets with different levels of crude glycerin. We used 105 male Cobb broilers, reared under intensive starter diets from the initial phase until termination, with slaughter at 42 days. The design was completely randomized (DIC) with five treatments and seven replicates $(5 \times 7)$, and each plot consisted of three birds. The treatments were as follows: T1 - basal diet without addition of glycerin, T2 - 30 $\mathrm{g} / \mathrm{kg}$ of adding glycerin, T3 $-60 \mathrm{~g} / \mathrm{kg}$ of adding glycerin, T4 $-90 \mathrm{~g} / \mathrm{kg}$ of adding glycerin and T5 $-120 \mathrm{~g} / \mathrm{kg}$ by addition of glycerin. The results showed the 
effect of levels of glycerin on the carcass parameters (Slaughter Weight, Carcass Yield and Breast, Weight Legs and Wings). For the color of breast meat, increases the levels of red and change the hue angle of color. No changes were observed in relation to weight loss by cooking, ultimate $\mathrm{pH}$ and tenderness in both cuts. The crude glycerin did not promote changes in values of nutrient composition in chicken meat regardless of the level of use and cut. Overall, the crude glycerin in the diet of chickens despite affecting carcass, stands out as an ingredient of potential use due to the small changes observed in relation to meat quality.

Keywords: softness, color, animal breeding, nutrition

\section{Introdução}

A busca por combustíveis alternativos ao petróleo transformou-se em objetivo comum de centros de pesquisa em todo o mundo nos últimos anos. 0 Brasil, por exemplo, é um dos países com mais resultados nesta área e utiliza há décadas o álcool combustível em automóveis. Neste sentido, o biodiesel, nome genérico dado a combustíveis e aditivos derivados de fontes renováveis, como óleos vegetais e gorduras animais, tornou-se uma aposta para o futuro.

O crescimento da produção de biocombustíveis tem gerado um excedente de glicerina, dificultando a absorção deste subproduto no mercado e o seu uso na alimentação animal pode ser uma alternativa interessante, não só na redução do custo das dietas, mas também, para contribuir com a sustentabilidade da produção de biodiesel.

Para a produção de aves, a alimentação é um dos fatores de maior custo no sistema produtivo devido à grande utilização de soja e milho na composição das rações. A glicerina ser uma importante fonte de glicerol (80 a 902\%) para produção de energia dietética através da lipogênese e gliconeogênese (Cerrate et al., 2006; Dozier et al., 2008; Lammers et al., 2008) a sua inclusão na dieta de frangos pode levar a ocorrência de comportamentos diferenciados em relação ao desenvolvimento muscular, uma vez que, existe diferenças 
relacionadas à proporção dos tipos de fibras musculares presentes em cada corte; atividade de enzimas relacionadas ao seu metabolismo como a glicerol quinase e proteina carreadoras como as aquagliceroporinas (Maeda et al., 2008).

Os experimentos em geral com uso de glicerina na alimentação de frangos, não tem mostrado alterações em relação ao desempenho e características de carcaça das aves em diferentes níveis de utilização (Simon et al., 1996 e Silva, 2010), revelando uma boa perspectiva para uso desse ingrediente, enquanto estudos referentes à qualidade de carne ainda são escassos.

O objetivo desse estudo é verificar a influência da utilização da glicerina bruta sobre os parâmetros de carcaça e qualidade de carne de frangos criados em sistema intensivo.

\section{Material e Métodos}

O estudo foi desenvolvido em parceria do Instituto Federal de Educação, Ciência e Tecnologia de Mato Grosso - IFMT Campus São Vicente e a Universidade Federal de Lavras - UFLA.

O experimento a campo foi conduzido na fazenda do IFMT Campus São Vicente localizado na serra de São Vicente da Serra em Santo Antônio do Leverger-MT. O experimento foi realizado nas instalações do Galpão Experimental de Avicultura do setor de Zootecnia I. As dietas foram formuladas de acordo com recomendações de Rostagno et al. (2005). O percentual de inclusão da glicerina foi de $0,30,60,90$ e $120 \mathrm{~g} / \mathrm{kg}$ da matéria natural da dieta (Tabela 1 e 2). As dietas foram fornecidas em todas as fases de criação: inicial, a partir do $1^{\circ}$ até $21^{\circ}$ dias e, crescimento/terminação de $22^{\circ}$ a $42^{\circ}$ dia. Após esse período, as aves foram abatidas para realização das avaliações de qualidade de carne. 
FARIA, P.B. et al. Qualidade de carcaça e carne de frangos com uso de glicerina na alimentação. PUBVET, Londrina, V. 7, N. 24, Ed. 247, Art. 1631, Dezembro, 2013.

Tabela 1 - Composição centesimal e valores calculados das dietas experimentais na Fase Inicial

\begin{tabular}{|c|c|c|c|c|c|}
\hline \multirow{2}{*}{ Ingredientes } & \multicolumn{5}{|c|}{ Níveis de glicerina ( $\mathrm{g} / \mathrm{kg}$ de matéria natural) } \\
\hline & 0 & 30 & 60 & 90 & 120 \\
\hline Milho triturado & 518,9 & 485,6 & 452,2 & 418,9 & 399,4 \\
\hline Farelo de soja & 375,3 & 381,4 & 387,4 & 393,4 & 385,6 \\
\hline Glicerina bruta ${ }^{1}$ & 0 & 30,0 & 60,0 & 90,0 & 120,0 \\
\hline Gordura suina & 45,0 & 42,3 & 39,7 & 37,0 & 34,3 \\
\hline Fosfato bicálcico & 2,76 & 2,84 & 2,92 & 2,91 & 3,09 \\
\hline Sal $(\mathrm{NaCl})$ & 1,82 & 1,83 & 1,84 & 1,86 & 1,87 \\
\hline $\begin{array}{l}\text { Premix vitamínico e } \\
\text { mineral }\end{array}$ & 50,0 & 50,0 & 50,0 & 50,0 & 50,0 \\
\hline DL-Metionina 99 & 2,78 & 2,82 & 2,87 & 2,91 & 2,96 \\
\hline L-Lisir & 2,88 & 2,71 & 2,54 & 2,38 & 2,21 \\
\hline L-Treonina 98 & 0,56 & 0,56 & 0,55 & 0,54 & 0,54 \\
\hline \multicolumn{6}{|l|}{ Valores calculados } \\
\hline $\begin{array}{l}\text { Energia Metabolizável } \\
(\text { KCal/kg) }\end{array}$ & 2,9804 & 2,9804 & 2,9804 & 2,9804 & 2,9804 \\
\hline Lisina digestível $(\mathrm{g} / \mathrm{kg})$ & 12,100 & 12,100 & 12,100 & 12,100 & 12,100 \\
\hline $\begin{array}{l}\text { Metionina + cisteina } \\
\text { digestível }(\mathrm{g} / \mathrm{kg})\end{array}$ & 8,600 & 8,600 & 8,600 & 8,600 & 8,600 \\
\hline Proteína bruta $(\mathrm{g} / \mathrm{kg})$ & 215,0 & 215,0 & 215,0 & 215,0 & 215,0 \\
\hline Treonina digestível $(\mathrm{g} / \mathrm{kg})$ & 7,80 & 7,80 & 7,80 & 7,80 & 7,80 \\
\hline Arginina digestivel $(\mathrm{g} / \mathrm{kg})$ & 13,879 & 13,951 & 14,024 & 14,097 & 14,170 \\
\hline Fósforo disponível (g/kg) & 4,600 & 4,600 & 4,600 & 4,600 & 4,600 \\
\hline Sódio (g/kg) & 2,20 & 2,20 & 2,20 & 2,20 & 2,20 \\
\hline Valina digestivel $(\mathrm{g} / \mathrm{kg})$ & 9,060 & 9,060 & 9,059 & 9,059 & 9,059 \\
\hline $\begin{array}{l}\text { Triptofano digestível } \\
(\mathrm{g} / \mathrm{kg})\end{array}$ & 2,413 & 2,427 & 2,441 & 2,454 & 2,468 \\
\hline Cálcio (g/kg) & 11,231 & 11,256 & 11,282 & 11,307 & 11,333 \\
\hline \multicolumn{6}{|c|}{ 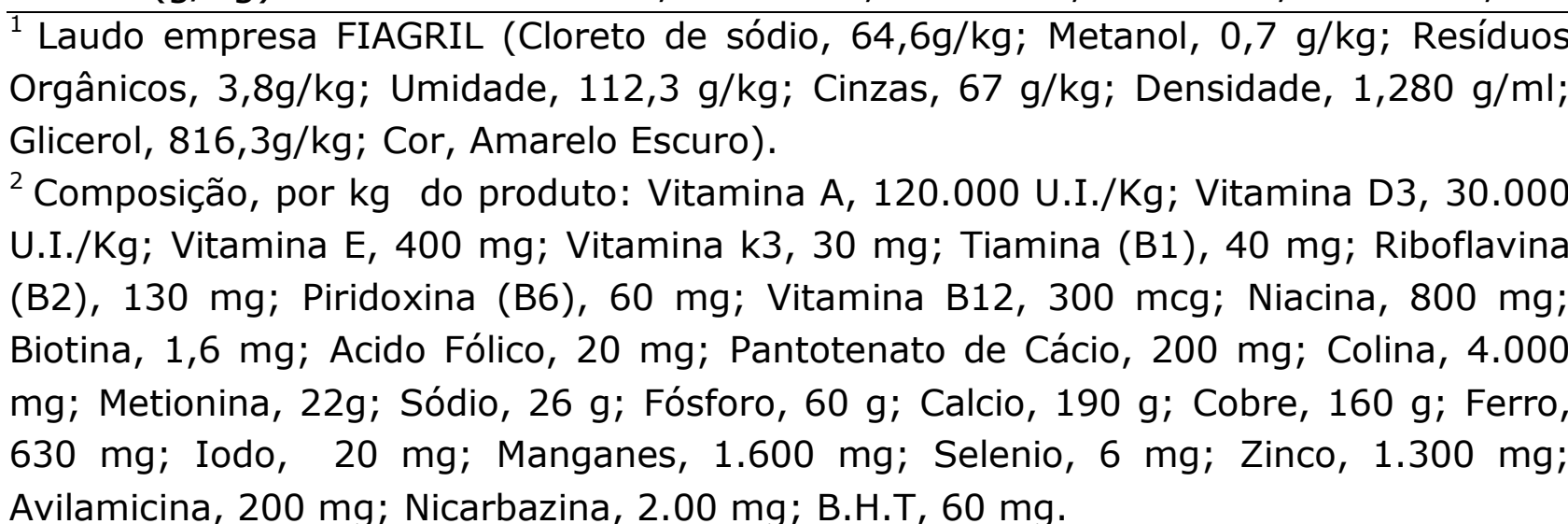 } \\
\hline
\end{tabular}


FARIA, P.B. et al. Qualidade de carcaça e carne de frangos com uso de glicerina na alimentação. PUBVET, Londrina, V. 7, N. 24, Ed. 247, Art. 1631, Dezembro, 2013.

Tabela 2 - Composição centesimal e valores calculados das dietas experimentais na fase de crescimento/terminação

\begin{tabular}{|c|c|c|c|c|c|}
\hline \multirow{2}{*}{ Ingredientes } & \multicolumn{5}{|c|}{ Níveis de glicerina ( $\mathrm{g} / \mathrm{kg}$ de matéria natural) } \\
\hline & 0 & 30 & 60 & 90 & 120 \\
\hline Milho triturado & 550,24 & 516,84 & 483,52 & 450,19 & 416,87 \\
\hline Farelo de soja & 316,85 & 322,88 & 328,91 & 334,93 & 340,96 \\
\hline Glicerina bruta ${ }^{1}$ & 0 & 30 & 60 & 90 & 120 \\
\hline Gordura suina & 70,00 & 67,38 & 64,71 & 62,04 & 59,38 \\
\hline Fosfato bicálcico & 5,35 & 5,43 & 5,52 & 5,61 & 5,69 \\
\hline Sal $(\mathrm{NaCl})$ & 1,43 & 1,44 & 1,45 & 1,47 & 1,48 \\
\hline $\begin{array}{l}\text { Premix vitamínico e } \\
\text { mineral }^{2}\end{array}$ & 50,00 & 50,00 & 50,00 & 50,00 & 50,00 \\
\hline DL-Metionina 99 & 2,42 & 2,46 & 2,50 & 2,55 & 2,59 \\
\hline L-Lisina $50 \%$ & 3,13 & 2,96 & 2,79 & 2,63 & 2,46 \\
\hline L-Treonina 98 & 0,60 & 0,59 & 0,59 & 0,58 & 0,58 \\
\hline \multicolumn{6}{|l|}{ Valores calculados } \\
\hline $\begin{array}{l}\text { Energia Metabolizável } \\
\text { (KCal/kg) }\end{array}$ & 3,1548 & 3,155 & 3,155 & 3,155 & 3,155 \\
\hline Lisina digestível $(\mathrm{g} / \mathrm{kg})$ & 10,80 & 10,80 & 10,80 & 10,80 & 10,80 \\
\hline $\begin{array}{l}\text { Metionina + cisteina } \\
\text { digestível }(\mathrm{g} / \mathrm{kg})\end{array}$ & 7,7 & 7,7 & 7,7 & 7,7 & 7,7 \\
\hline Proteína bruta (g/kg) & 190,9 & 190,9 & 190,9 & 190,9 & 190,9 \\
\hline Treonina digestível $(\mathrm{g} / \mathrm{kg})$ & 7,00 & 7,00 & 7,00 & 7,00 & 7,00 \\
\hline Fósforo disponível (g/kg) & 5,00 & 5,00 & 5,00 & 5,00 & 5,00 \\
\hline Sódio (g/kg) & 2,04 & 2,04 & 2,04 & 2,04 & 2,04 \\
\hline Cálcio $(\mathrm{g} / \mathrm{kg})$ & 11,736 & 11,761 & 11,787 & 11,812 & 11,838 \\
\hline
\end{tabular}

${ }^{1}$ Laudo empresa FIAGRIL (Cloreto de sódio, 64,6g/kg; Metanol, 0,7 g/kg; Resíduos Orgânicos, 3,8g/kg; Umidade, 112,3 g/kg; Cinzas, $67 \mathrm{~g} / \mathrm{kg}$; Densidade, 1,280 g/ml; Glicerol, 816,3g/kg; Cor, Amarelo Escuro).

${ }^{2}$ Composição, por kg do produto: Vitamina A, 120.000 U.I./Kg; Vitamina D3, 30.000 U.I./Kg; Vitamina E, 400 mg; Vitamina k3, 30 mg; Tiamina (B1), 40 mg; Riboflavina (B2), 130 mg; Piridoxina (B6), 60 mg; Vitamina B12, 300 mcg; Niacina, 800 mg; Biotina, 1,6 mg; Acido Fólico, 20 mg; Pantotenato de Cácio, 200 mg; Colina, 4.000 mg; Metionina, 22g; Sódio, $26 \mathrm{~g}$; Fósforo, $60 \mathrm{~g}$; Calcio, $190 \mathrm{~g}$; Cobre, $160 \mathrm{~g}$; Ferro, 630 mg; Iodo, 20 mg; Manganes, 1.600 mg; Selenio, 6 mg; Zinco, 1.300 mg; Avilamicina, $200 \mathrm{mg}$; Nicarbazina, $2.00 \mathrm{mg}$; B.H.T, $60 \mathrm{mg}$. 
Para avaliação dos aspectos de qualidade de carne, foram utilizadas um total de 105 frangos (cento e cinco frangos) machos da linhagem Cobb, amostrados aleatoriamente de cada boxe. O Delineamento foi Inteiramente Casualizado (DIC), com cinco tratamentos (Tratamento 1 - ração basal sem adição de glicerina; Tratamento $2-30 \mathrm{~g} / \mathrm{kg}$ de adição de glicerina em substituição ao milho da ração basal; Tratamento 3 - $60 \mathrm{~g} / \mathrm{kg}$ de adição de glicerina em substituição ao milho da ração basal; Tratamento $4-90 \mathrm{~g} / \mathrm{kg}$ de adição de glicerina em substituição ao milho da ração basal e, Tratamento 5 $120 \mathrm{~g} / \mathrm{kg}$ de adição de glicerina em substituição ao milho da ração basal) e 7 (sete) repetições por tratamento, sendo cada parcela experimental constituída por 3 (três) aves.

O abate das aves foi realizado aos 42 dias, nas dependências do frigorífico existente no IFMT Campus São Vicente (Santo Antonio do Leverger-MT) através de método humanitário e uso de boas práticas de higiene. Os frangos, após o abate, foram pesados e embalados individualmente em sacos plásticos. As aves, em seguida ao abate, foram resfriadas em câmara fria, onde permaneceram por 24 horas à temperatura de $5^{\circ} \mathrm{C}$. Nas carcaças dos frangos, 24 horas post mortem, foram coletadas amostras do músculo do peito (Pectoralis major) e da coxa que foram encaminhados congelados para Universidade Federal de Lavras - UFLA para realização das análises físicoquímicas e de composição bromatológica.

As carcaças dos frangos foram consideradas a ave sem pescoço e sem pé. Após 24 horas post mortem, estas foram divididas em cortes comerciais: peito, pernas (coxa + sobre-coxa), dorso, pescoço e asas, sendo estes pesados de acordo com cada unidade experimental ( 3 aves).

As análises físico-químicas foram realizadas no Laboratório de Tecnologia de Carnes e Pescado do Departamento de Ciência dos Alimentos da UFLA. As análises foram realizadas após 24 horas de descongelamento da amostras em geladeira. As determinações de $\mathrm{pH}$ foram realizadas com potenciômetro DIGIMED DM-20 acoplado a um eletrodo, sendo tomadas nos cortes peito e coxa. Para cor, foi utilizado colorímetro MINOLTA CR 200b (Osaka - Japão), 
operando no sistema CIEL*a*b*, com iluminante D65, calibrado em padrão branco ladrilho. Para a cor ainda foi determinados os índices de saturação (C*) e o ângulo de tonalidade $(h *)$, que foram calculados pelas seguintes fórmulas (Ramos \& Gomide, 2007): $C^{*}=(a * 2+b * 2) 1 / 2$ e; $h *=\tan -1(b * / a *)$.

Após as determinações de cor, foi realizada a perda de peso por cocção (PPC). Para PPC as amostras foram pesadas em balança semi-analítica, METTLER M P1210 (Toledo, Brasil), envolvidas em papel alumínio e submetidas a cozimento em chapa elétrica à $150^{\circ} \mathrm{C}$ até ultrapassar $72^{\circ} \mathrm{C}$ (Amasa, 1978). Para determinação da maciez foi utilizada metodologia de Froning \& Uijttenboogarte (1988), onde as amostras foram amostradas em ambos os cortes no sentido longitudinal da fibra $(1,0 \times 1,0 \times 4,0 \mathrm{~cm})$ e posteriormente seccionadas no sentido transversal das fibras musculares, utilizando uma sonda Warner Bratzler acoplada a um Texturômetro modelo TA XT-2.

As amostras para a análise proximal foram preparadas com a retirada da pele, tecido adiposo e aponeuroses, seguida de homogeneização. As análises de umidade, proteína, extrato etéreo e cinzas foram realizadas conforme Horwitz (2000), em duplicata.

Os dados foram analisados com apoio do programa SISVAR $R$. As variáveis com respostas de efeitos significativos na análise de variância (Teste F) foram submetidas análise de Regressão $(a=0,05)$.

\section{Resultados e Discussão}

O uso de glicerina na alimentação dos frangos mostrou efeito sobre os parâmetros de carcaça avaliados $(P<0,05)$, com modificação nos valores de peso em jejum, peso das pernas e asas e rendimento de carcaça e peito (Tabela 03). 
FARIA, P.B. et al. Qualidade de carcaça e carne de frangos com uso de glicerina na alimentação. PUBVET, Londrina, V. 7, N. 24, Ed. 247, Art. 1631, Dezembro, 2013.

Tabela 3 - Composição da carcaça de frangos em função do nível de glicerina na alimentação.

\begin{tabular}{|c|c|c|c|c|c|c|c|}
\hline \multirow[t]{2}{*}{ Parâmetros } & \multicolumn{5}{|c|}{$\begin{array}{l}\text { Nível de inclusão de glicerina ( } \mathrm{g} / \mathrm{kg} \text { de matéria } \\
\text { natural) }\end{array}$} & \multirow{2}{*}{$\begin{array}{l}\text { CV } \\
(\%)^{1}\end{array}$} & \multirow{2}{*}{$\begin{array}{l}\text { Valor } \\
\text { de } \mathrm{P}^{2}\end{array}$} \\
\hline & 0 & 30 & 60 & 90 & 120 & & \\
\hline $\begin{array}{l}\text { Peso Jejum } \\
\text { (g) }\end{array}$ & 3089,29 & 2941,43 & 3044,52 & 2917,62 & 2847,62 & 4,12 & 0,0058 \\
\hline Carcaça (g) & 2295,71 & 2145,04 & 2193,94 & 2256,82 & 2218,83 & 4,29 & 0,0577 \\
\hline $\begin{array}{l}\text { Rend. } \\
\text { Carcaça } \\
(\mathrm{g} / \mathrm{kg})\end{array}$ & 74,32 & 73,08 & 72,03 & 77,52 & 78,32 & 4,29 & 0,0029 \\
\hline Pescoço (g) & 51,74 & 46,90 & 45,83 & 49,57 & 50,60 & 10,71 & 0,2046 \\
\hline $\begin{array}{l}\text { Rend. } \\
\text { Pescoço } \\
(\mathrm{g} / \mathrm{kg})\end{array}$ & 2,24 & 2,16 & 2,10 & 2,19 & 2,31 & 10,70 & 0,5338 \\
\hline Peito (g) & 904,40 & 826,61 & 883,43 & 898,03 & 885,48 & 6,67 & 0,1302 \\
\hline $\begin{array}{l}\text { Rend. Peito } \\
(\mathrm{g} / \mathrm{kg})\end{array}$ & 39,10 & 37,87 & 40,14 & 39,68 & 40,16 & 3,62 & 0,0290 \\
\hline Dorso (g) & 436,69 & 431,28 & 419,37 & 445,00 & 420,25 & 4,95 & 0,1470 \\
\hline $\begin{array}{l}\text { Rend. Dorso } \\
(\mathrm{g} / \mathrm{kg})\end{array}$ & 18,90 & 19,82 & 19,10 & 19,70 & 19,09 & 4,37 & 0,1912 \\
\hline Perna (g) & 678,50 & 626,40 & 628,44 & 635,99 & 641,31 & 4,25 & 0,0079 \\
\hline $\begin{array}{l}\text { Rend. Perna } \\
(\mathrm{g} / \mathrm{kg})\end{array}$ & 29,37 & 28,80 & 28,67 & 28,13 & 29,15 & 3,22 & 0,1429 \\
\hline Asas (g) & 224,40 & 213,85 & 216,87 & 228,23 & 221,19 & 3,65 & 0,0174 \\
\hline $\begin{array}{l}\text { Rend. Asas } \\
(\mathrm{g} / \mathrm{kg})\end{array}$ & 9,72 & 9,84 & 9,90 & 10,12 & 10,06 & 3,23 & 0,1444 \\
\hline
\end{tabular}

Os resultados mostraram alteração no peso de abate das aves com o uso de glicerina na alimentação (Tabela 04). A ração com nível de glicerina de 60 $\mathrm{g} / \mathrm{kg}$ promoveu menor efeito sobre o valores de peso de abate, com redução de 1,45\%, enquanto nos demais níveis, houve redução de 4,79\% (30 g/ $\mathrm{kg})$, $5,56(90 \mathrm{~g} / \mathrm{kg})$ e $7,82 \%(120 \mathrm{~g} / \mathrm{kg})$. Silva (2010), apesar de não encontrar efeito significativo do valores de inlusão de glicerina no peso vivo das aves aos 
42 dias, observou que ao nível de inclusão de $100 \mathrm{~g} / \mathrm{kg}$, houve redução de $4,2 \%$. Cerrate et al. (2006), relatou comportamento semelhante com redução $5,75 \%$ com inclusão de $100 \mathrm{~g} / \mathrm{kg}$ de glicerina na dieta.

A avaliação dos aspectos físico-químicos revelou influência dos níveis de glicerina sobre os índices de cor no peito, alterando os teores de vermelho ( $a^{*}$ ) e o ângulo de tonalidade da cor ( $\mathrm{h} *$ ) (Tabela 04).

Os diferentes níveis de glicerina na dieta não influenciaram os valores de composição centesimal dos cortes peito e coxa, corroborando os achados de Guerra (2010), que até o nível de inclusão de $100 \mathrm{~g} / \mathrm{kg}$ de glicerina na carne de frangos não encontraram diferenças para os valores de matéria seca, proteína, extrato etéreo e cinzas. Estes resultados estão próximos aos relatados por Garcia et al. (2005) e Faria et al. (2008) para frangos comerciais e; Murakami et al. (2010), que trabalhando com diferentes fontes lipídicas, encontraram valores de lipídeos totais semelhantes aos valores verificados neste trabalho para carne do peito da linhagem Cobb.

Os valores de umidade e cinzas em ambos os cortes com uso da glicerina foram semelhantes, somente havendo diferença entres eles em relação aos teores de proteína e extrato etéreo. Esse comportamento é verificado normalmente na carne de aves, em vista das características dos cortes em relação a sua composição tecidual (Tipos de fibras, deposição de tecido adiposo, metabolismo) sendo independente de fatores nutricionais, genéticos ou sistema de produção (Castellini et al., 2002; Faria et al., 2008; Faria et al., 2009). 
FARIA, P.B. et al. Qualidade de carcaça e carne de frangos com uso de glicerina na alimentação. PUBVET, Londrina, V. 7, N. 24, Ed. 247, Art. 1631, Dezembro, 2013.

Tabela 4 - Composição físico-química e centesimal dos cortes Peito e Coxa de frangos em função do nível de glicerina na alimentação.

\begin{tabular}{|c|c|c|c|c|c|c|c|c|}
\hline \multirow[t]{2}{*}{ Corte } & \multirow[t]{2}{*}{ Parâmetros } & \multicolumn{5}{|c|}{$\begin{array}{l}\text { Nível de inclusão de glicerina ( } \mathrm{g} / \mathrm{kg} \text { da } \\
\text { matéria natural) }\end{array}$} & \multirow[t]{2}{*}{$\begin{array}{l}\mathrm{CV} \\
(\%)^{1}\end{array}$} & \multirow[t]{2}{*}{$\begin{array}{l}\text { Valor de } \\
\qquad P^{2}\end{array}$} \\
\hline & & 0 & 30 & 60 & 90 & 120 & & \\
\hline \multirow{12}{*}{ Peito } & $L^{*}$ & 48,15 & 47,95 & 48,44 & 48,45 & 47,87 & 3,56 & 0,9512 \\
\hline & a* & 4,58 & 4,75 & 4,55 & 5,26 & 5,86 & 13,57 & 0,0042 \\
\hline & $b^{*}$ & 6,98 & 6,77 & 6,60 & 6,91 & 5,66 & 17,00 & 0,1941 \\
\hline & PPC $(\mathrm{g} / 100 \mathrm{~g})$ & 21,89 & 20,29 & 19,50 & 23,09 & 22,96 & 20,93 & 0,4865 \\
\hline & FC (kgf) & 1,50 & 1,46 & 1,56 & 1,68 & 1,84 & 17,34 & 0,1080 \\
\hline & $\mathrm{pH}$ & 5,95 & 5,94 & 5,89 & 5,90 & 5,90 & 1,70 & 0,7583 \\
\hline & $C^{*}$ & 8,39 & 8,30 & 8,07 & 8,73 & 8,18 & 11,31 & 0,7323 \\
\hline & $\mathrm{h} *$ & 56,45 & 54,78 & 54,96 & 52,55 & 43,72 & 11,96 & 0,0051 \\
\hline & $\begin{array}{l}\text { Umidade } \\
(\mathrm{g} / 100 \mathrm{~g})\end{array}$ & 75,08 & 74,67 & 74,95 & 75,74 & 75,01 & 1,14 & 0,2305 \\
\hline & $\begin{array}{l}\text { Proteína } \\
\text { (g/100g) }\end{array}$ & 21,53 & 20,66 & 21,81 & 21,76 & 20,48 & 7,97 & 0,4354 \\
\hline & $\begin{array}{l}\text { Cinzas } \\
(\mathrm{g} / 100 \mathrm{~g})\end{array}$ & 0,99 & 1,01 & 1,13 & 1,02 & 1,09 & 10,55 & 0,1274 \\
\hline & $\begin{array}{l}\text { E. Etéreo } \\
(\mathrm{g} / 100 \mathrm{~g})\end{array}$ & 2,03 & 2,10 & 2,05 & 1,92 & 1,94 & 28,84 & 0,9733 \\
\hline \multirow{12}{*}{ Coxa } & $L^{*}$ & 49,80 & 48,01 & 49,28 & 47,97 & 47,04 & 4,07 & 0,0912 \\
\hline & $a^{*}$ & 9,18 & 10,58 & 9,79 & 9,52 & 11,07 & 15,76 & 0,1782 \\
\hline & $b^{*}$ & 7,49 & 7,71 & 7,37 & 7,20 & 6,61 & 15,50 & 0,4422 \\
\hline & PPC $(\mathrm{g} / 100 \mathrm{~g})$ & 27,16 & 24,83 & 27,01 & 24,00 & 28,36 & 16,14 & 0,3091 \\
\hline & $F C(K g f)$ & 1,28 & 1,30 & 1,18 & 1,32 & 1,43 & 16,81 & 0,3430 \\
\hline & $\mathrm{pH}$ & 6,25 & 6,18 & 6,26 & 6,27 & 6,30 & 1,58 & 0,2415 \\
\hline & $\mathrm{C}^{*}$ & 11,86 & 13,13 & 12,30 & 12,09 & 12,92 & 11,67 & 0,4375 \\
\hline & $\mathrm{h}^{*}$ & 39,06 & 36,25 & 36,92 & 37,89 & 30,96 & 17,59 & 0,1803 \\
\hline & $\begin{array}{l}\text { Umidade } \\
(\mathrm{g} / 100 \mathrm{~g})\end{array}$ & 76,16 & 75,97 & 76,05 & 75,69 & 76,02 & 1,62 & 0,9656 \\
\hline & $\begin{array}{l}\text { Proteína } \\
\text { (g/100g) }\end{array}$ & 17,41 & 19,50 & 18,26 & 17,72 & 18,57 & 11,08 & 0,3591 \\
\hline & $\begin{array}{l}\text { Cinzas } \\
(\mathrm{g} / 100 \mathrm{~g})\end{array}$ & 0,89 & 1,03 & 1,01 & 1,01 & 0,95 & 18,91 & 0,6022 \\
\hline & $\begin{array}{l}\text { Extrato Etéreo } \\
(\mathrm{g} / 100 \mathrm{~g})\end{array}$ & 4,89 & 4,01 & 4,21 & 4,52 & 4,63 & 30,25 & 0,7615 \\
\hline
\end{tabular}

${ }^{1}$ Coeficiente de Variação. ${ }^{2}$ - Probabilidade: Teste F. 
Não foi observado efeito dos níveis de glicerina nos valores de $\mathrm{pH}$ final do peito e da coxa (Tabela 4). Almeida et al. (2002) estudando dietas de frango de corte, não constataram influência dos tratamentos sobre os valores de $\mathrm{pH}$ do peito, indicando que nutrição não apresentou influência direta sobre esse parâmetro. No presente estudo, os valores de $\mathrm{pH}$ final do peito foram inferiores aos da coxa. Faria et al. (2009), encontraram comportamento semelhante, com médias de pH na coxa acima de 6,00. Esse comportamento em função do corte, segundo Erickson (1998) pode estar relacionado a uma maior concentração de mioglobina, devido a uma maior proporção de fibras vermelhas nos músculos e, como conseqüência, ocorrência de uma menor capacidade de armazenamento de glicogênio. O que pode ser evidenciado devido ao maior valor de a* encontrado para coxa, corroborando os resultados de Garcia et al. (2005) que relataram redução conjunta dos valores de vermelho e $\mathrm{pH}$.

$\mathrm{Na}$ análise da coxa, não foram encontradas diferenças nos parâmetros de cor em função dos níveis de glicerina na dieta (Tabela 4). Garcia et al (2005) avaliando índice de luminosidade da coxa, também não encontraram influência com a substituição do milho na ração. Alguns trabalhos (Pérez-Vendrell, 2001; Garcia et al., 2005) mostram que a substituição do milho por outros ingredientes pode influenciar o teor de amarelo da carne, estando esta modificação relacionada a redução da quantidade de pigmentos carotenóides na alimentação. Entretanto, neste estudo o teor de amarelo não diferiu entre os tratamentos, nao mostrando redução com aumento do nível de glicerina na dieta. Este resultado foi semelhante ao encontrado para frangos machos da linhagem Cobb por Santos et al. (2005), que relaram média de 5,98. Esse comportamento pode ser devido a propria caracteristicas fisico-quimica da glicerina que apresentava a cor amarelo escuro, o que pode ter contribuido para que os pigmentos presentes na sua composição nao interferissem no índice de amarelo da carne do peito e coxa.

Em ambos os cortes, não foi observado efeito dos tratamentos sobre os valores de perda de peso por cozimento (PPC), confirmando os resultados de 
FARIA, P.B. et al. Qualidade de carcaça e carne de frangos com uso de glicerina na alimentação. PUBVET, Londrina, V. 7, N. 24, Ed. 247, Art. 1631, Dezembro, 2013.

Garcia et al. (2005), que não encontraram efeito do uso da substituição do milho pelo sorgo na ração de frangos de corte. As médias de PPC para peito variaram de 19,50 a 23,09g/100g e não mostraram efeito do diferentes níveis de glicerina. Estes valores são semelhante aos descritos por Santos et al. (2005) que encontraram média de $21,69 \mathrm{~g} / 100 \mathrm{~g}$ e; inferiores aos citados por Bressan \& Beraquet (2004), que relatam médias para peito de frango de 28,44 a 29,03g/100g e de Souza et al. (2011) que citam média de 29,63g/100g para linhagem Cobb.

A perda de peso por cozimento da coxa variou de 24,00 a $28,36 \mathrm{~g} / 100 \mathrm{~g}$ (Tabela 4). Esses valores foram inferiores aos valores relatados por Souza et al. (2011), que citaram média de PPC de 34,11g/100g para coxa de frangos Cobb. Este comportamento pode ter ocorrido devido aos menores valores de $\mathrm{pH}(5,96)$ encontrado por estes autores, uma vez que a capacidade de retenção de água da carne depende da evolução do $\mathrm{pH}$ e quanto mais baixos esses valores, menor é a quantidade água retida no músculo, implicando em maior exsudação de líquidos (Price \& Schweigert, 1994).

Os valores de FC no peito foram inferiores aos apresentados por Pavan et al. (2003) que descreveram médias de 1,91 a 2,23 kgf; Bressan \& Beraquet (2004) de 2,92 a 3,50 kgf e, por Souza et al. (2011) de 2,09 kgf para frangos da linhagem Cobb. Entretanto, até o nível de $120 \mathrm{~g} / \mathrm{kg}$ de glicerina não foi verificado alteração na maciez da carne independente do corte, o que discorda dos resultados de Garcia et al. (2005) que encontraram aumento do valor de FC em relação ao nível de substituição do milho pelo sorgo na carne do peito. Os valores de força de cisalhamento da carne da coxa e do peito foram bem similares e não mostraram influência dos tratamentos, sendo consideradas bem macias (Souza et al., 2011).

Os resultados mostraram uma redução do peso de abate em função do nível glicerina (Tabela 05), apesar de não haver efeito significativo sobre o peso da carcaça. Contudo, para o rendimento de carcaça, ocorreu um comportamento quadrático $(P<0,05)$, indicando que 0 uso da glicerina pode proporcionar em relação as concentrações utilizadas, um comportamento 
FARIA, P.B. et al. Qualidade de carcaça e carne de frangos com uso de glicerina na alimentação. PUBVET, Londrina, V. 7, N. 24, Ed. 247, Art. 1631, Dezembro, 2013.

diferenciado, com incrementos de rendimento nos níveis de $90 \mathrm{~g} / \mathrm{kg}(4,31 \%)$ e $120 \mathrm{~g} / \mathrm{kg}(5,38 \%)$. Cerrate et al. (2006) relataram redução do rendimento de carcaça ao nível de $100 \mathrm{~g} / \mathrm{kg}$ de glicerina na ração, resultado que somente foi observado neste trabalho nos níveis de $30 \mathrm{~g} / \mathrm{kg}(-1,67 \%)$ e $60 \mathrm{~g} / \mathrm{kg}(-3,08 \%)$. Entretanto, Silva (2010) nao encontrou resultado significativo sobre os valores de rendimento de carcaça, com o uso de diferentes níveis de glicerina na dieta de frangos.

Para rendimento do corte peito, houve aumento linear com o uso da glicerina na alimentação (Tabela 05). Cerrate et al. (2006) e Silva (2010), verificaram resultados semelhante e, segundo estes autores, este comportamento pode ter ocorrido devido ao potencial gliconeogênico do glicerol, uma vez que, neste músculo há um grande número de fibras glicolíticas (Smith \& Fletcher, 1988), favorecendo o seu maior desenvolvimento. Considerando estes aspectos, para o peso da perna (coxa + sobre-coxa) foi verificado efeito quadrático, com redução dos valores até o nível de $71,31 \mathrm{~g} / \mathrm{kg}$ de glicerina na dieta. Esse comportamento pode ter ocorrido em virtude da maior frequência de fibras intermediárias (FOG) e oxidativas (SO) (Madeira et al., 2006; e Ono et al. (1993), onde glicerol estaria sendo utilizado para produção de energia via ciclo de Krebs, uma vez que a quantidade de mitocôndrias é abundante nestes músculos, o que não influenciou proporcionalmente o rendimento deste corte.

Por outro lado, para o peso das asas, os dados mostraram melhor ajustamento para função cúbica (Tabela 05), apesar de não ser verificado influência sobre os valores de rendimento.

Para carne do peito, foi verificado que os níveis de glicerina, ocasionaram redução do ângulo de tonalidade ( $h *$, enquanto não ocorreu modificação do índice de saturação (C*), (Tabela 5). Brown et al. (2008) relataram para carne do peito de frangos Cobb, valores de 4,2 para C* e, de 54,7 para h* e, segundo estes autores, a alimentação com a presença de pigmentos carotenóides pode influenciar esses índices, o que não foi observado neste trabalho. 
Tabela 5 - Parâmetros de peso, carcaça, corte e cor de frangos em função do nível de glicerina na alimentação.

\begin{tabular}{|c|c|c|c|c|c|}
\hline \multirow{2}{*}{ Variáveis } & \multirow{2}{*}{ Equação } & \multicolumn{4}{|c|}{ Valor $\mathrm{P}^{12}$} \\
\hline & & L & $\mathrm{Q}$ & C & FA \\
\hline $\begin{array}{l}\text { Peso ao } \\
\text { abate }\end{array}$ & $\hat{Y}=3069,5240-1,690476 \times X$ & 0,002 & 0,670 & 0,194 & 0,288 \\
\hline & $r^{2}=0,6715$ & & & & \\
\hline $\begin{array}{l}\text { Rendimento } \\
\text { Carcaça }\end{array}$ & $\begin{array}{c}\hat{Y}=74,079714-0,059586 \times X+ \\
0,000842 \times X^{2} \\
r^{2}=0,7761\end{array}$ & 0,003 & 0,027 & 0,216 & 0,975 \\
\hline $\begin{array}{l}\text { Rendimento } \\
\text { Peito }\end{array}$ & $\begin{array}{c}\hat{Y}=38,605429+0,013062 \times X \\
r^{2}=0,4216\end{array}$ & 0,029 & 0,731 & 0,143 & 0,056 \\
\hline Peso Perna & $\begin{array}{c}\hat{Y}=672,278490-1,362237 \times X+ \\
0,009552 \times X^{2} \\
r^{2}=0,8098\end{array}$ & 0,056 & 0,004 & 0,094 & 0,095 \\
\hline Peso Asa & $\begin{array}{c}\hat{Y}=224,703755-0,883295 \times X+ \\
0,018977 \times X^{2}-0,000099 \times X^{3} \\
r^{2}=0,9501\end{array}$ & 0,415 & 0,188 & 0,002 & 0,035 \\
\hline a* peito & $\begin{array}{c}\hat{Y}=4,382000+0,010262 \times X \\
r^{2}=0,7550\end{array}$ & 0,001 & 0,074 & 0,760 & 0,288 \\
\hline $\mathrm{h} *$ peito & $\begin{array}{c}\hat{Y}=58,029429-0,092262 \times X \\
r^{2}=0,7373\end{array}$ & 0,001 & 0,067 & 0,279 & 0,975 \\
\hline
\end{tabular}

${ }^{1}$ L, Q e C: efeitos de ordem linear, quadrática e cúbica relativos à inclusão de glicerina na dieta. ${ }^{2} \mathrm{FA}$ : Falta de Ajustamento

O teor de vermelho $\left(a^{*}\right)$ na carne do peito aumentou de forma linear em função do nível de glicerina na alimentação dos frangos (Tabela 5). Esse comportamento pode ser resultado de um aumento na quantidade de mioglobina, uma vez que o glicerol atua como agente da gliconeogênese e na inibição das atividades das enzimas fosfoenolpiruvato carboxiquinase e glutamato desidrogenase, o pode resultar na economia dos aminoácidos gluconeogênicos (Cerrate et al. 2006). Apesar do predomínio de fibras glicoliticas no peito e que apresentam baixa atividade enzimática oxidativa e metabolismo energético glicolítico (Forrest et al.,1979; Ono et al., 1993)), esse 
maior aporte de glicerol que é convertido em glicerol-3-fosfato, seria utilizado pelas mitocôndrias para fosforilação oxidativa e produção de energia. Desta forma, este aumento na atividade oxidativa, seria acompanhado por uma

maior quantidade de mitocôndria e consequentemente de citocromo, o que teria ocasionado o aumento na cor vermelho do músculo em vista destas proteínas possuírem grupos prostéticos com a presença de ferro na sua estrutura (Nelson \& Cox, 2008).

Os resultados indicam que a carne do peito apresentou uma de forma geral uma tonalidade de cor laranja e que o aumento do nível de glicerina na dieta promoveu uma maior tendência para o vermelho. Esse comportamento esta relacionado ao aumento do teor de vermelho nos maiores nível de inclusão de glicerina na dieta, uma vez que, o ângulo de tonalidade é dependente das variações e $a *$ e $b^{*}$, sendo representado através de equação linear (Tabela 5).

De forma geral era esperado que o fornecimento da glicerina bruta na alimentação das aves ocasionasse um aumento nos teores de lipídeos na carne ou redução da catálise de aminoácidos e maior acúmulo de proteína no músculo, favorecendo conjuntamente a retenção de proteína corporal (Cerrate et al. 2006). Entretanto, neste trabalho não foi verificado alteração desses parâmetros, o que pode ser devido ao fato das dietas serem isoproteicas e isoenergéticas e a glicerina apresentar um valor energético semelhante ao milho.

\section{Conclusão}

O uso da glicerina bruta na alimentação de frangos de corte promove alterações dos parâmetros de carcaça, com aumento na cor vermelha na carne do peito em maiores níveis na alimentação. 


\section{Referências}

ALMEIDA, J. C. L.; MENDES, A. A.; GARCIA, R. G. et al. Efeito do nível de lisina da dieta e do sexo sobre o desempenho e rendimento de carcaça de frango de corte. Rev. Bras. Ciênc. Avíc., v. 4, n. 1, p.11-18, 2002.

AMASA. Guidelines for cooking sensory evaluation of meat. American Meat Science Association, National Live Stock and Meat Board:Chicago, 1978. 24p.

BRESSAN, M. C.; BERAQUET, N. J. Tratamentos de pré resfriamento e resfriamento sobre a qualidade de carne de peito de frango. Ciênc. e Tecnol Aliment., v. 24, n. 2, p. 230-235, 2004.

BROWN, S. N.; NUTE, G. R.; BAKER, A. et al. Aspects of meat and eating quality of broiler chickens reared under standard, maize-fed, free-range or organic systems. Br. Poult. Sci., v. 49, n. 2, p.118-124, 2008.

CASTELLINI, C.; MUGNAI, C.; DAL BOSCO, A. Effect of organic production system on broiler carcass and meat quality. Meat Sci., v. 60, n. 3, p. 219-225, 2002.

CERRATE, S., YAN, F.; WANG, Z. et al. Evaluation of glycerine from biodiesel production as a feed ingredient for broilers. Int. J. Poult. Sci., v.5, n.11, p.1001-1007, 2006.

DOZIER, W.A.; KERR, B.J.; CORZO, A. et al. Apparent metabolizable energy of glycerin for broiler chickens. Poult. Sci., v.87, n.2, p.317-322, 2008.

ERICKSON, H. H. Fisiologia do Exercício. In: Dukes, M.J.S. Fisiologia dos animais domésticos. Rio de Janeiro, Guanabara Koogan, 11.ed., p.277-296, 1998.

FARIA P.B.; BRESSAN, M.C.; SOUZA, X.R. et al. Composição proximal e qualidade da carne de frangos das linhagens Paraíso Pedrês e Pescoço Pelado. Rev. Bras. Zoot., v. 38, n. 12, p. 2455-2464, 2009.

FARIA, P. B.; NETO, J. V.; BRESSAN, M. C. et al. Qualidade da carne de marreco Pequim branco (Anas Platyrhynchos platyrhynchos L.1758) comparado a frango de corte. Ciênc. Agrotec., v. 32, n. 1, p.213-218, 2008.

FAROUK, M.M.; PRICE, J. F. The effect of post-exsanguination infusion on the composition, exudation, color and post-mortem metabolic changes in lamb. Meat Sci., v.38, n.3, p.477-496, 1994.

FORREST, J.C.; ABERLE, E.D.; HEDRICK, H.B. et al. Fundamentos de ciencia de la carne. Zaragoza. Ed. Acribia, 1979. 364 p.

FRONING, G. W.; UIJTTENBOOGAART, T. G. Effect of post mortem electrical stimulation on color, texture, $\mathrm{pH}$ and cooking loses of hot and cold deboned chicken broiler breast meat. Poult. Sci., v. 67, n. 11, p. 1536-1544, 1988.

GARCIA, R. G. MENDES, A. A.; COSTA, C. et al. Desempenho e qualidade da carne de frangos de corte alimentados com diferentes níveis de sorgo em substituição ao milho. Arq. Bras. Med. Vet. Zoot., v.57, n.5, p.634-643, 2005.

GUERRA, R. L. H. Glicerina bruta na alimentação de frangos de Corte. Dissertação (Mestrado em Zootecnia) - Universidade Estadual de Maringá, Maringá. 2010. 55f.

HORWITZ, W. (ed.). Official methods of analysis of the association of official analytical chemists, Gaithersburg Maryland, USA: AOAC International, 17th ed., 2000.

MADEIRA, L. A.; SARTORI, J. R.; SALDANHA, E. S. P. B.; et al. Morfologia das fibras musculares esqueléticas de frangos de corte de diferentes linhagens criados em sistema de confinamento e semiconfinamento. Rev. Bras. Zoot., v. 35, n. 6, p. 2322-2332, 2006. 
MAEDA, N.; FUNAHASHI, T.; SHIMOMURA I. Metabolic impact of adipose andhepatic glycerol channels aquaporin 7 and aquaporin 9. Nat. Clin. Pract. Endocrinol MeTabela, v.4, n.11, p.627634, 2008.

MOUROT, J.; AUMAITRE, A.; MOUNIER, A. et al. Nutritional and physiological effects of dietary glycerol in the growing pig. Consequences on fatty tissues and post mortem muscular parameters. Livest. Prod. Sci., v. 38, n.3, p. 237-244, 1994.

NELSON, D. L.; COX, M. M. Lehninger Principles of Biochemistry. 5th ed. W. H. Freeman: New York, 2008. 1100p.

ONO, Y.; IWAMOTO, H; TAKAHARA, H. The relationship between muscle growth and the growth of different fiber types in the chicken. Poult. Sci., v. 72, p.548-576, 1993.

PAVAN, A. C.; MENDES, A. A.; OLIVEIRA, E. G. Efeito da linhagem e do nível de lisina da dieta sobre a qualidade da carne do peito de frangos de corte. Rev. Bras. Zoot., v.32, n.6, suppl.1, p.1732-1736, 2003.

PÉREZ-VENDRELL, A.M. Influence of source and ratio of xanthophyll pigments on broiler chicken pigmentation and performance. Poult. Sci., v.80, p.320-326, 2001.

PRICE, J. F.; SCHWEIGGERT, B. S. Ciência de la carne y de los productos carnicos. Zaragoza: Acribia, 1976. 668p.

RAMOS, E.M.; GOMIDE, L.A.M. Avaliação da Qualidade de Carnes: fundamentos e metodologias. Viçosa, MG. Ed. UFV, 2007. 599p.

ROSTAGNO, H.S.; ALBINO, L.F.T.; DONZELE, J.L. et al. Tabelas brasileiras para aves e suínos, composição de alimentos e exigências nutricionais. Viçosa, Ed. UFV. 2aa, 2005.186p.

SANTOS, A. L.; SKOMURA, N. K.; FREITAS, E. R. et al. Estudo do crescimento, desempenho, rendimento de carcaça e qualidade de carne de três linhagens de frango de corte. Rev. Bras. Zoot., v. 34, n. 5, p. 1589-1598, 2005.

SILVA, C. L. S. Glicerina proveniente da produção de biodiesel como ingrediente de ração para frangos de corte. Dissertação (Mestrado em Zootecnia) Escola Superior de Agricultura "Luiz de Queiroz"/Universidade de São Paulo, Piracicaba. 2010. 81f.

SIMON, A.; BERGNER, H.; SCHWABE, M. Glicerol - feed ingredient for broiler chickens. Arch. Anim. Nutr., v.49, n.2, p.103-112, 1996.

SMITH, D. P.; FLETCHER, D. L. Chicken breast muscle fiber type and diameter as influenced by age and intramuscular location. Poult. Sci., v. 67, n.6, p. 908-913, 1988. 